\title{
Effect of maternal pre-pregnancy BMI and weekly gestational weight gain on the development of infants
}

\author{
Chao Li', Lingxia Zeng ${ }^{1}$, Duolao Wang ${ }^{2}$, Shaonong Dang ${ }^{1}$, Tao Chen², Victoria Watson ${ }^{2}$ and Hong Yan ${ }^{1,3,4,5^{*}}$
}

\begin{abstract}
Objective: The aim of the present study is to identify the average effect across different time points and to specify the time effects of maternal pre-pregnancy BMI and weekly gestational weight gain on the mental development and physical growth of infants.

Methods: The present cohort study used a repeated measures study design that began in 2004 with follow up at $3,6,12,18$, and 24 months of age. The participants were a subset from a controlled, cluster-randomized, doubleblind trial. Bayley Scales of Infant Development (BSID) were used to estimate the mental development of infants. A generalized estimating equation linear model was used to estimate the effects of maternal BMl and weight gain.

Results: The average effect of maternal BMI and weight gain on the weight for age $Z$ scores (WAZ), length for age $Z$ scores (LAZ) and mental development index (MDI) across the different time points of infants was significant. In addition, the maternal BMI and weight gain were positively and significantly associated with the WAZ and LAZ in infants of different ages. However, the effect of weekly gestational weight gain was significant only during the earlier period of life (3 months, Coefficient: 11.15, 95\%Cl: 4.89-17.41).

Conclusions: Our results indicate positive effects of pre-pregnancy and prenatal nutrition on the physical growth of infants. Weekly gestational weight gain of the pregnant women had a positive effect on the mental development of the infants, but this effect appears to decline over time.
\end{abstract}

Keywords: Maternal pre-pregnancy BMI, Weekly gestational weight gain, Development of infants

\section{Introduction}

The intellectual development and physical growth in the early life are important for future health and wellbeing. Many prospective studies indicated that mental development was significantly associated with further school achievement, motor and cognitive performance and leadership success and was inversely associated with several health outcomes ascertained in later life [1-4]. Evidence from a series of population-based cohort studies indicated that physical growth in infancy affects the further total and abdominal fat distribution in school-aged

\footnotetext{
* Correspondence: yh.paper_xjtu@aliyun.com

'Department of Epidemiology and Biostatistics, School of Public Health, Xi'an Jiaotong University Health Science Center, Xi'an, China

${ }^{3}$ Nutrition and Food Safety Engineering Research Center of Shaanxi Province, Xi'an, China

Full list of author information is available at the end of the article
}

children as well as the proximal femoral geometry in adulthood $[5,6]$.

Recently, the effects of maternal BMI and weight gain on child development have been well studied in higher resource settings. For example, evidence from a cohort study conducted in the United States showed a significant association between the maternal pre-pregnancy BMI and body size of infants at 6 months of age [7]. Another US cohort study found a positive effect of maternal weight gain during pregnancy on the prevalence of overweight children 3 years of age [8]. A large prospective, population-based birth cohort study conducted in the United Kingdom reported a significant association between the maternal pre-pregnancy BMI and children's cognitive performance [9]. Another UK cohort study reported that the gestational weight gain was positively associated with the school entry assessment scores of 
children at 4 years of age [10]. However, there is a lack of robust data on the effects of maternal BMI and weight gain in a lower resource setting where there are concerns that inadequate in utero nutrition may have adverse effects on children's development. In addition, the comprehensive evaluation of the average effect on the mental development and physical growth of infants has rarely been studied.

Therefore, the present cohort study conducted in Changwu and Bing County, which used a repeated measures study design, provides prospective data to identify the average effect across different time points and to specify the time effect of maternal pre-pregnancy BMI and weekly gestational weight gain on the mental development and physical growth of infants (3-24 months).

\section{Methods}

\section{Study design and population}

The present study was a subset of a larger cluster-randomized, double blind trial conducted between 2002 and 2006 in the counties of Changwu and Bing in Shaanxi Province, China. Singleton neonates born only in 2004 were included in the present study. The details of this large trial have been described elsewhere [11-13]. The primary aim of this large trial was to compare the differences in birth outcomes (birthweight and neonatal mortality) among the prenatal micronutrient supplementation groups. The prenatal micronutrient supplementation groups were multi-micronutrient (15 minerals or vitamins), folic acid plus iron, and folic acid (control group). The pregnant women from the same village received the same treatment (prenatal micronutrient supplementation) from enrolment until delivery. The RCT was conducted in Changwu and Bing counties, and there were 14 townships and 234 villages in Changwu County and 20 townships and 327 villages in Bing County included in the study. The randomization of villages was stratified by county with a fixed ratio of treatment (1:1:1) and blocking of 15 to ensure geographical balance with an approximately equal distribution of treatments per township.

The baseline information (sociodemographic, menstrual, reproductive, and medical status, and family history) was collected in the large trial. In addition, the pregnant women enrolled in the trial received three free prenatal care checks at different stages of pregnancy, at which the physical examination including blood pressure and weight measurement was undertaken by trained maternal and child health staff. There were 4604 single live births in the trial, and 1305 of the births occurred in 2004.

Due to limited funding, a subgroup of singleton neonates (born in 2004) was included in the present cohort study and were followed up at 3, 6, 12, 18 and 24 months of age. The baseline characteristics among the participants delivered in 2004 and others were compared, and there were no differences in the baseline characteristics (data not shown). A face-to-face interview was conducted with the primary carers to collect information about the morbidity of infants, time spent outdoors per day and feeding practices. The length of the study was specifically designed with the primary goal of detecting moderate differences in the mean mental development index (MDI) among the three prenatal micronutrient supplementation groups. However, this sample was considered sufficient for detecting 5 IQ point differences, which were considered clinically significant between the prenatal micronutrient supplementation groups $[14,15]$.

\section{Setting}

The present study was conducted in Changwu and Bing counties, which are both situated in the western part of the Shaanxi Province, and the two counties' total area is approximately $1752 \mathrm{~km}^{2}$. In 2007 , the total population of the two rural counties was approximately 497,000 . The agricultural annual per capita net income was only 1249 yuan and 780 yuan in Bing and Changwu counties, respectively. The two counties were also classified as Type 4 (poorest) rural counties.

\section{Measurement}

\section{Outcomes}

During all visits, the anthropometric measurements and mental development (MD) tests were conducted. The weight and length of the infants were measured using standardized methods. Weight was measured to the nearest $10 \mathrm{~g}$ on an electronic scale, excluding the weight of infants' clothes. The scales were checked by calibration with a standard weight $(10 \mathrm{~kg})$ at regular $1 \mathrm{~mm}$ intervals on the length board. The length for age $Z$ scores (LAZ), weight for age $Z$ scores (WAZ) and weight for length $\mathrm{Z}$ scores (WLZ) of infants were calculated using the 2006 WHO reference values [16].

For the MD test, we used the Bayley Scales of Infant Development (BSID), which can be applied to infants between one and 42 months to assess the mental development of this subgroup of infants [17]. The BSID is a global measure of developmental status in infancy according to assessments of the timely attainment of relatively crude infant milestones [18]. The complete BSID consists of 178 mental scale items regarding sensory acuity, discrimination, memory, and early verbal communication [17, 19]. In the present study, the BSID, which was translated into Chinese, was standardized to be culturally appropriate. The reliability and validity of these measures have been evaluated and shown to be 
satisfactory $[13,20]$. The BSID is a standardized test, and a specific start and stop item were set depending on the age and developmental level of the infant [21]. The MD test was administered in the local hospital or the infant's own home when the infant was not sleepy, restless, or hungry. The examiners had been trained rigorously and demonstrated high levels of consistency with one another [13]. The raw MD scores were scored by the items that the infant passed on the MD scales of the BSID. The raw MD scores could be converted into an MDI by using standard procedures that are based on the norms of Chinese urban children.

\section{Exposures}

In the original large trial, recruited pregnant women could receive three prenatal health checks for free, at which they were asked about complications during pregnancy and were also weighted at the clinic by trained maternal and child health $(\mathrm{MCH})$ staff. The results from a Caucasian study indicated that maternal weight did not change in the first trimester, and changes in maternal weight during pregnancy usually occur after the first trimester [22]. Therefore, maternal BMI during the first trimester is being used as a proxy for pre-pregnancy BMI in the present study. Pre-pregnancy BMI was categorized as underweight $(\mathrm{BMI}<18.5)$, normal-weight $(18.5 \leq \mathrm{BMI}<25)$, overweight $(25 \leq \mathrm{BMI}<30)$, or obese $(B M I \geq 30)$ [23]. The total gestational weight gain was calculated from the maximum weight measured in the third trimester (the last prenatal health check) and the maternal weight was measured in the first trimester $(0-$ 14 weeks of gestational age). The gestational age used to calculate the weekly gestational weight gain was calculated from the weeks of gestation in the last prenatal health check in the third trimester minus the weeks of gestation in the first trimester. The weekly gestational weight gain was calculated from the total gestational weight gain divided by the gestational age. The gestational age at birth was measured as completed days based on the first day of the last menstrual period. In the present study, we excluded the participants who were not weighed in the first trimester. Of the 1305 women, 1073 women were weighted in the first trimester, and 1184 women were weighted in the third trimester (Fig. 1).

\section{Statistical analysis}

All the data were double-entered for verification and checked manually for completeness. Range, extremum, and logical checks were also conducted for accuracy. Statistical significance was set at a $P$ value $<0.05$ for all statistical tests, and testing was two-sided. The baseline information in the different pre-pregnancy nutritional groups was described by means, standard deviations or percentages. The baseline characteristics of the children and households were compared across the maternal pre-pregnancy nutritional status groups by using ANOVA, chi-square test or fisher exact test. In addition, a wealth index was generated from 16 different household assets or facilities with a principal component analysis method, and this index in the present study was divided into thirds as indicators for the richest, middle income, and poorest households. Due to the repeated measures structure of the data and the original randomized design, the generalized estimating equation (GEE) linear model was used to estimate the effect of maternal pre-pregnancy BMI and weekly gestational weight gain on the physical growth and mental development during the 3-24 months of infant growth. Meanwhile, an interaction between the maternal pre-pregnancy BMI, weekly gestational weight gain and time points of the repeated measurements (months) was added to the models to evaluate the subsequent results at the different time points of the repeated measurements. Estimates of the coefficients and $95 \%$ confidence intervals (CIs) were made.

Previous studies found that social factors and prenatal multi-micronutrient supplementation detrimentally affected the physical growth and mental development of children [24-28]. In addition, previous studies reported that higher weight before pregnancy results in less weight gain during pregnancy [29]. Therefore, separate models were fitted to study the effects of maternal weight and weight gain on child development: the model for maternal BMI was not adjusted for maternal weight gain as gestational weight gain is a downstream consequence of maternal weight status, while the model for weekly gestational weight gain was adjusted for maternal pre-pregnancy BMI.

Two models were fitted with multivariable analysis when estimating the effect of maternal pre-pregnancy BMI, with one model adding socio-economic variables (county, sex of infant, maternal age, educational level of parents, household wealth index and occupation of parents) and the other model including the variables of the gestational weeks at birth, gestational weeks when maternal weight was measured, type of prenatal micronutrient supplementation and doses of supplements consumed. When estimating the effect of weekly gestational weight gain, we added one more variable (maternal pre-pregnancy BMI) into the models. In addition, we added another interaction term (prenatal treatment groups and weekly gestational weight gain) into the model as a modification of the effect of the relationship between the gestational weight gain and intellectual development.

To reduce bias and improve the efficiency of the analysis, we used multiple imputation, which has become 


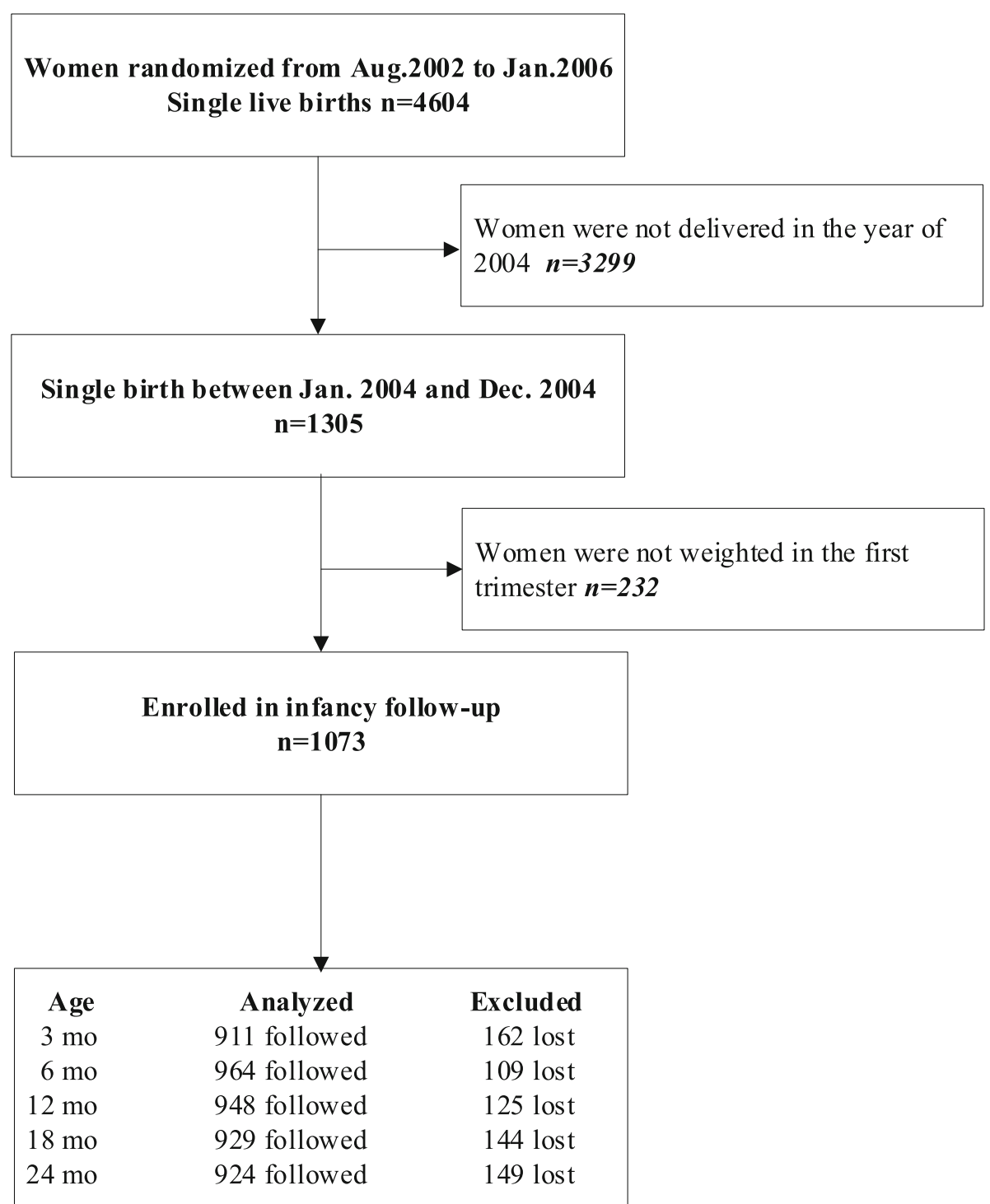

Fig. 1 Participant flow chart

increasingly popular for handling missing data to estimate the effect of maternal pre-pregnancy BMI and weekly gestational weight gain [30,31]. The differences between the results of the original GEE model and the imputation model were carefully examined. The data were analysed using STATA software, version 14.0 (Stata Corp LP, College Station, Texas, USA).

\section{Results}

The profile of this cohort study is shown in Fig. 1. In the present study, we excluded infants (17.8\%) whose mothers were not weighted in their first trimester. After doing so, the participants in and those excluded from the study did not differ in baseline characteristics (Additional file 1: Table S1). In addition, there were 162 (15.1\%), 109 (10.2\%), 125 (11.6\%), 144 (13.4\%), and 149
(13.9\%) infants who were lost to follow-up monitoring at 3 months, 6 months, 12 months, 18 months and 24 months, respectively.

Table 1 shows the baseline characteristics of the infants and households in the different maternal pre-pregnancy nutritional status groups. The prevalence of maternal overnutrition (overweight and obesity) and underweight were 2.7\% (overweight: 26/1073; obesity: 3/1073) and $17.7 \%$ (190/1073), respectively. For the characteristics of children, the highest means of the WAZ, LAZ, WLZ and MDI were in the maternal overweight/obesity group. Compared to the maternal underweight group, the WAZ, WLZ and LAZ of the infants were higher in the maternal normal BMI group. Women who were underweight had the highest weekly weight gain during pregnancy $(P=$ 0.003). In addition, the household economic status was 
Table 1 Baseline characteristics of children and households by maternal pre-pregnancy nutritional status ${ }^{\mathrm{a}}$

\begin{tabular}{|c|c|c|c|c|}
\hline & \multicolumn{4}{|l|}{ Maternal pre-pregnancy BMI } \\
\hline & $\begin{array}{l}\text { Underweight }(\mathrm{BMI}<18.5) \\
n=190\end{array}$ & $\begin{array}{l}\text { Normal }(18.5 \leq \mathrm{BMI}<25) \\
n=854\end{array}$ & $\begin{array}{l}\text { Overweight/Obesity }(\mathrm{BMl} \geq 25) \\
n=29\end{array}$ & $P$ \\
\hline \multicolumn{5}{|l|}{ Child characteristics } \\
\hline Gender & & & & 0.143 \\
\hline Boy & 109(57.4) & $527(61.7)$ & 22(75.9) & \\
\hline Girl & $81(42.6)$ & $327(38.3)$ & $7(24.1)$ & \\
\hline \multicolumn{5}{|l|}{ At the age of 3 months } \\
\hline WAZ & $0.43 \pm 1.03$ & $0.64 \pm 1.05$ & $1.11 \pm 1.01$ & 0.005 \\
\hline LAZ & $-0.51 \pm 1.10$ & $-0.42 \pm 1.12$ & $0.01 \pm 0.99$ & 0.109 \\
\hline WLZ & $1.16 \pm 1.19$ & $1.38 \pm 1.24$ & $1.54 \pm 0.96$ & 0.080 \\
\hline MDI & $107.53 \pm 10.94$ & $107.92 \pm 11.24$ & $109.31 \pm 14.55$ & 0.764 \\
\hline \multicolumn{5}{|l|}{ At the age of 6 months } \\
\hline WAZ & $0.37 \pm 1.10$ & $0.69 \pm 1.01$ & $1.25 \pm 0.96$ & $<0.001$ \\
\hline LAZ & $-0.82 \pm 1.24$ & $-0.64 \pm 1.21$ & $-0.16 \pm 1.27$ & 0.035 \\
\hline WLZ & $1.23 \pm 1.24$ & $1.50 \pm 1.14$ & $1.82 \pm 1.41$ & 0.010 \\
\hline MDI & $93.02 \pm 16.82$ & $93.54 \pm 15.42$ & $94.15 \pm 17.85$ & 0.907 \\
\hline \multicolumn{5}{|l|}{ At the age of 12 months } \\
\hline WAZ & $0.27 \pm 0.96$ & $0.42 \pm 1.01$ & $1.05 \pm 1.10$ & 0.002 \\
\hline LAZ & $-0.64 \pm 1.18$ & $-0.52 \pm 1.09$ & $0.22 \pm 1.01$ & 0.002 \\
\hline WLZ & $0.76 \pm 1.02$ & $0.89 \pm 1.08$ & $1.25 \pm 1.11$ & 0.095 \\
\hline MDI & $103.26 \pm 21.92$ & $102.16 \pm 21.80$ & $104.71 \pm 21.22$ & 0.731 \\
\hline \multicolumn{5}{|l|}{ At the age of 18 months } \\
\hline WAZ & $0.17 \pm 0.89$ & $0.34 \pm 0.90$ & $0.71 \pm 0.88$ & 0.011 \\
\hline LAZ & $-0.83 \pm 1.12$ & $-0.81 \pm 1.09$ & $-0.38 \pm 0.78$ & 0.165 \\
\hline WLZ & $0.78 \pm 0.91$ & $0.96 \pm 0.97$ & $1.19 \pm 0.97$ & 0.045 \\
\hline MDI & $99.90 \pm 19.91$ & $99.55 \pm 19.89$ & $106.30 \pm 19.43$ & 0.235 \\
\hline \multicolumn{5}{|l|}{ At the age of 24 months } \\
\hline WAZ & $0.22 \pm 0.91$ & $0.24 \pm 0.90$ & $0.75 \pm 0.79$ & 0.023 \\
\hline LAZ & $-0.62 \pm 1.20$ & $-0.64 \pm 1.02$ & $-0.38 \pm 0.71$ & 0.492 \\
\hline WLZ & $0.87 \pm 0.95$ & $0.93 \pm 0.97$ & $1.43 \pm 1.01$ & 0.032 \\
\hline $\mathrm{MDI}$ & $89.05 \pm 20.44$ & $90.44 \pm 17.79$ & $93.18 \pm 14.37$ & 0.499 \\
\hline Gestational age at birth, wk. & $39.92 \pm 1.38$ & $39.93 \pm 1.51$ & $39.94 \pm 1.47$ & 0.996 \\
\hline \multicolumn{5}{|l|}{ Women's characteristics } \\
\hline Maternal age, y & $24.06 \pm 4.09$ & $24.83 \pm 4.43$ & $25.46 \pm 4.96$ & 0.078 \\
\hline Weekly gestational weight gain, kg/wk & $0.39 \pm 0.25$ & $0.34 \pm 0.19$ & $0.27 \pm 0.18$ & 0.003 \\
\hline Women's education & & & & 0.018 \\
\hline Primary & $41(21.6)$ & $256(30.0)$ & $9(31.0)$ & \\
\hline Secondary & 119(62.6) & $476(55.9)$ & $20(69.0)$ & \\
\hline$\geq$ High school & $30(15.8)$ & $120(14.1)$ & 0 & \\
\hline Women's occupation at enrollment & & & & 0.180 \\
\hline Farmer & 159(83.7) & 713(83.9) & 28(96.6) & \\
\hline Others & $31(16.3)$ & 137(16.1) & $1(3.4)$ & \\
\hline Father's education & & & & 0.427 \\
\hline Primary & 19(10.1) & $90(10.6)$ & 0 & \\
\hline
\end{tabular}


Table 1 Baseline characteristics of children and households by maternal pre-pregnancy nutritional status ${ }^{\mathrm{a}}$ (Continued)

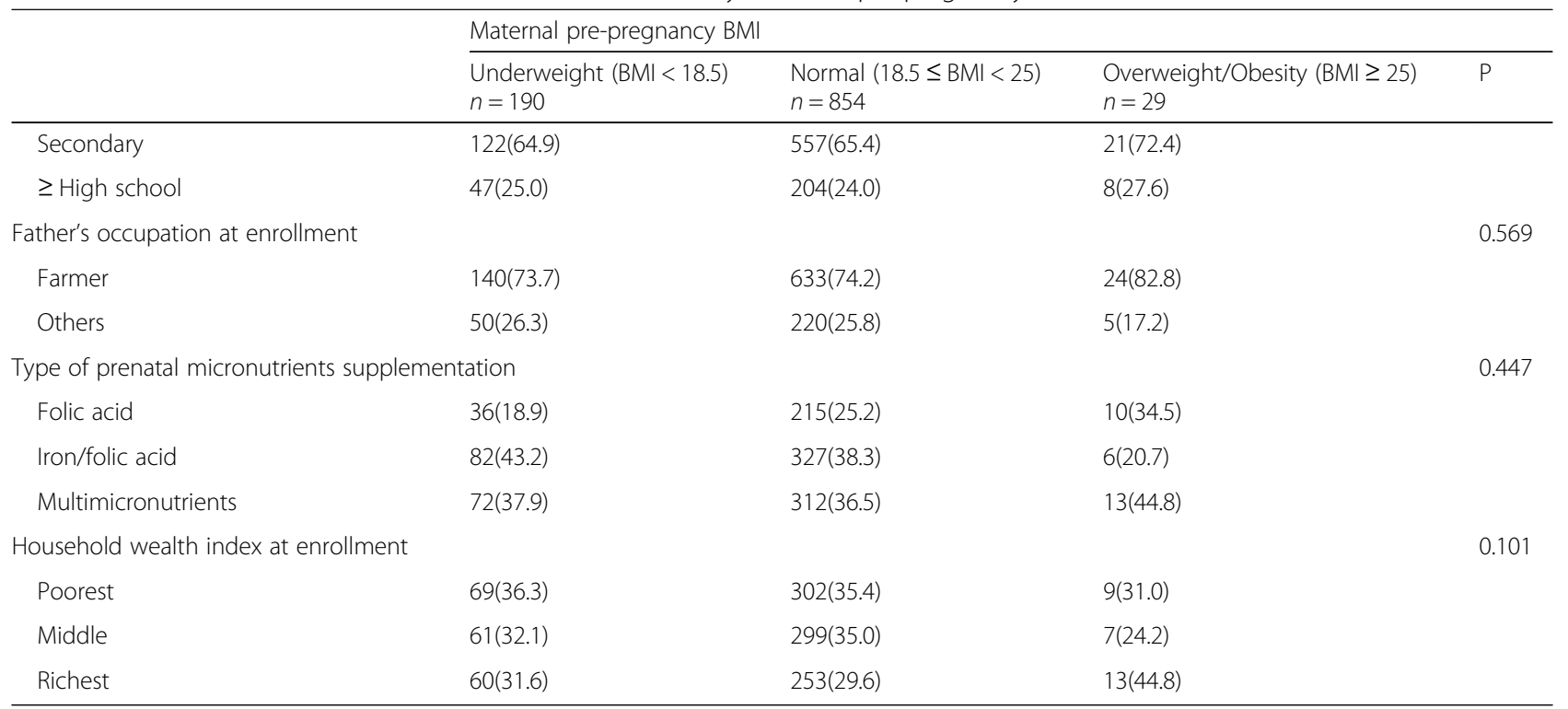

Abbreviation: LAZ Length for age Z scores, MDI Mental Development, WAZ Weight for age Z scores, WLZ Weight for length Z scores

avalues are $\mathrm{n}(\%)$ or means \pm SDs. Anova, Chi-square or Fisher exact test were used to compare differences among three groups

best in the women who were overweight or obese, but the difference was not significant $(P=0.101)$.

Table 2 shows the significant association between the maternal pre-pregnancy BMI and WAZ (total, Coefficient: 0.07, 95\%CI: 0.04-0.09), LAZ (total, Coefficient: 0.03 , 95\%CI: $0.01-0.06$ ) and WLZ (total, Coefficient: 0.06, 95\%CI: 0.04-0.08) of infants, but the association between the pre-pregnancy BMI and MDI (total, Coefficient: 0.22 , 95\%CI: -0.11-0.54) was not significant. In the unadjusted analysis, the maternal pre-pregnancy BMI was significantly associated with the infant WAZ and WLZ at the ages of 3, 6, 12, 18 and 24 months. However, the LAZ was significantly associated with the maternal pre-pregnancy BMI at earlier ages of 3, 6 and 12 months. In addition, the pre-pregnancy BMI was associated with the infant WAZ (Coefficient: 11.15, 95\%CI: 4.89-17.41), LAZ (Coefficient: 11.15, 95\%CI: 4.89-17.41) and WLZ (Coefficient: 11.15, 95\%CI: 4.89-17.41). After adjusting for the variables of county, sex of infant, maternal age, educational level of parents, household wealth index, occupation of parents (Model 1), gestational weeks at birth, gestational weeks when maternal weight was measured, maternal pre-pregnancy BMI, type of prenatal micronutrient supplementation and doses of supplements consumed (Model 2), the effect of the maternal pre-pregnancy BMI was still significant in the different ages of the infants. For the mental development of infants, the association between the maternal pre-pregnancy BMI and MDI of the infants was not significant at any age $(3,6,12,18,24$ months). The average effect of the maternal pre-pregnancy BMI was significant on the physical growth of infants but not significant on the mental development of infants, and the results were similar among the unadjusted analysis models, model 1 and model 2. In addition, we found no material differences after adding an interaction term (prenatal treatment groups and weekly gestational weight gain) into the GEE model (Additional file 1: Table S2).

A similar analysis was conducted to estimate the relationships between the weekly gestational weight gain and physical growth (WAZ, LAZ and WLZ) and the mental development (MDI) of infants. Overall, there was a significant association between the weekly gestational weight gain and WAZ (Coefficient: 0.38, 95\%CI: 0.09-0.66) as well as the LAZ (Coefficient: 0.14, 95\%CI: 0.12-0.76) and MDI (Coefficient: 5.01, 95\%CI: $1.03-8.99)$ of infants, but the association between the weekly gestational weight gain and WLZ (Coefficient: 0.14, 95\%CI: -0.12-0.41) was not significant. For physical growth, the results from models 1 and 2 showed a significant association between the weekly gestational weight gain and physical growth (WAZ and LAZ) at different time points of measurement. For mental development, the effect of weekly gestational weight gain was significant in the earlier period of life (3 months, Coefficient: 11.15, 95\%CI: 4.89-17.41, Model 2). The average effect of weekly gestational weight gain across the different time points was significant in the WAZ (Coefficient: 0.38, 95\%CI: 0.09-0.66, Model 2) and LAZ (Coefficient: 0.44, 95\%CI: 0.12-0.76, Model 2) after controlling for the confounders. In addition, the average effects of 
Table 2 Association between maternal pre-pregnancy BMI and development of infants ${ }^{a}$

\begin{tabular}{|c|c|c|c|c|c|c|}
\hline & \multicolumn{6}{|c|}{ Maternal pre-pregnancy BMl } \\
\hline & \multicolumn{2}{|l|}{ Unadjusted analysis } & \multicolumn{2}{|l|}{ Model $1^{\mathrm{b}}$} & \multicolumn{2}{|l|}{ Model $2^{c}$} \\
\hline & Coef(95\%Cl) & $P$ & Coef( $95 \% C l)$ & $P$ & Coef $(95 \%$ Cl) & $P$ \\
\hline \multicolumn{7}{|c|}{ Age of 3 months } \\
\hline WAZ & $0.07(0.04,0.09)$ & $<0.001$ & $0.07(0.04,0.10)$ & $<0.001$ & $0.07(0.05,0.10)$ & $<0.001$ \\
\hline LAZ & $0.04(0.01,0.07)$ & 0.027 & $0.04(0.01,0.07)$ & 0.016 & $0.04(0.01,0.07)$ & 0.013 \\
\hline WLZ & $0.05(0.02,0.08)$ & 0.002 & $0.06(0.02,0.09)$ & 0.001 & $0.06(0.02,0.09)$ & 0.001 \\
\hline $\mathrm{MDI}$ & $0.34(-0.19,0.87)$ & 0.204 & $0.47(-0.06,1.00)$ & 0.085 & $0.39(-0.14,0.92)$ & 0.145 \\
\hline \multicolumn{7}{|c|}{ Age of 6 months } \\
\hline WAZ & $0.08(0.06,0.11)$ & $<0.001$ & $0.09(0.06,0.12)$ & $<0.001$ & $0.09(0.06,0.12)$ & $<0.001$ \\
\hline LAZ & $0.04(0.01,0.07)$ & 0.015 & $0.05(0.01,0.08)$ & 0.005 & $0.05(0.02,0.08)$ & 0.004 \\
\hline WLZ & $0.08(0.05,0.11)$ & $<0.001$ & $0.08(0.05,0.11)$ & $<0.001$ & $0.08(0.05,0.11)$ & $<0.001$ \\
\hline $\mathrm{MDI}$ & $-0.07(-0.59,0.44)$ & 0.785 & $0.13(-0.38,0.65)$ & 0.612 & $0.07(-0.45,0.58)$ & 0.796 \\
\hline \multicolumn{7}{|c|}{ Age of 12 months } \\
\hline WAZ & $0.05(0.02,0.08)$ & 0.001 & $0.06(0.03,0.08)$ & $<0.001$ & $0.06(0.03,0.08)$ & $<0.001$ \\
\hline LAZ & $0.04(0.01,0.07)$ & 0.021 & $0.04(0.01,0.07)$ & 0.012 & $0.04(0.01,0.08)$ & 0.009 \\
\hline WLZ & $0.04(0.01,0.07)$ & 0.008 & $0.04(0.01,0.08)$ & 0.007 & $0.04(0.01,0.08)$ & 0.006 \\
\hline $\mathrm{MDI}$ & $0.12(-0.39,0.64)$ & 0.638 & $0.35(-0.17,0.87)$ & 0.188 & $0.28(-0.24,0.80)$ & 0.297 \\
\hline \multicolumn{7}{|c|}{ Age of 18 months } \\
\hline WAZ & $0.06(0.03,0.09)$ & $<0.001$ & $0.06(0.03,0.09)$ & $<0.001$ & $0.06(0.04,0.09)$ & $<0.001$ \\
\hline LAZ & $0.01(-0.03,0.04)$ & 0.694 & $0.01(-0.02,0.04)$ & 0.565 & $0.01(-0.02,0.04)$ & 0.509 \\
\hline WLZ & $0.08(0.04,0.11)$ & $<0.001$ & $0.08(0.04,0.11)$ & $<0.001$ & $0.08(0.04,0.11)$ & $<0.001$ \\
\hline $\mathrm{MDI}$ & $0.01(-0.51,0.53)$ & 0.965 & $0.13(-0.39,0.65)$ & 0.625 & $0.06(-0.46,0.58)$ & 0.824 \\
\hline \multicolumn{7}{|c|}{ Age of 24 months } \\
\hline WAZ & $0.04(0.01,0.07)$ & 0.005 & $0.05(0.02,0.08)$ & 0.001 & $0.05(0.02,0.08)$ & 0.001 \\
\hline LAZ & $0.01(-0.02,0.04)$ & 0.530 & $0.01(-0.02,0.05)$ & 0.386 & $0.02(-0.02,0.05)$ & 0.342 \\
\hline WLZ & $0.05(0.02,0.08)$ & 0.002 & $0.05(0.02,0.08)$ & 0.002 & $0.05(0.02,0.08)$ & 0.002 \\
\hline $\mathrm{MDI}$ & $0.13(-0.39,0.66)$ & 0.620 & $0.35(-0.17,0.88)$ & 0.189 & $0.39(-0.24,0.81)$ & 0.281 \\
\hline \multicolumn{7}{|l|}{ Total } \\
\hline WAZ & $0.06(0.04,0.08)$ & $<0.001$ & $0.07(0.04,0.09)$ & $<0.001$ & $0.07(0.04,0.09)$ & $<0.001$ \\
\hline LAZ & $0.03(0.01,0.05)$ & 0.044 & $0.03(0.01,0.06)$ & 0.021 & $0.03(0.01,0.06)$ & 0.016 \\
\hline WLZ & $0.06(0.04,0.08)$ & $<0.001$ & $0.06(0.04,0.08)$ & $<0.001$ & $0.06(0.04,0.08)$ & $<0.001$ \\
\hline MDI & $0.10(-0.23,0.44)$ & 0.540 & $0.28(-0.04,0.61)$ & 0.089 & $0.22(-0.11,0.54)$ & 0.196 \\
\hline
\end{tabular}

Abbreviation: $C I$ confidence interval, $L A Z$ length for age $Z$ scores, MDI mental development index, WAZ weight for age $Z$ scores, WLZ weight for length Z scores

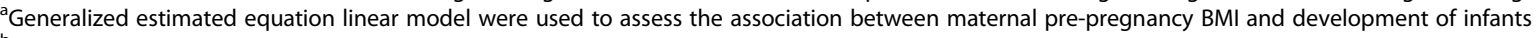

${ }^{\mathrm{b}}$ Model 1 include the variables of county, sex of infant, maternal age, educational level of parents, household wealth index, occupation of parents

'Model 2 include the variables of county, sex of infant, maternal age, educational level of parents, household wealth index, occupation of parents, gestational weeks at birth, gestational weeks when maternal weight measured, maternal pre-pregnancy BMI, type of prenatal micronutrient supplementation, doses of supplements consumed

weekly gestational weight gain were significant for the mental development (Coefficient: 5.01, 95\%CI: 1.03-8.99, Model 2) of infants. The results were similar among the unadjusted and multivariable analyses (Table 3). A sensitivity analysis was conducted using multiple imputation methods, and there were no material differences between the results of the sensitivity and original analyses (Additional file 1: Table S3, Additional file 1: Table S4).

\section{Discussion}

In the present study, we found the effects of maternal pre-pregnancy and weekly gestational weight gain on the physical and mental development of infants across different time points. In addition, the maternal pre-pregnancy BMI and weekly gestational weight gain were positively associated with the physical growth (WAZ and LAZ) of infants of different ages. The effect of the weekly gestational weight gain on the offspring's 
Table 3 Association between gestational weekly weight gain and development of infants ${ }^{\text {a }}$

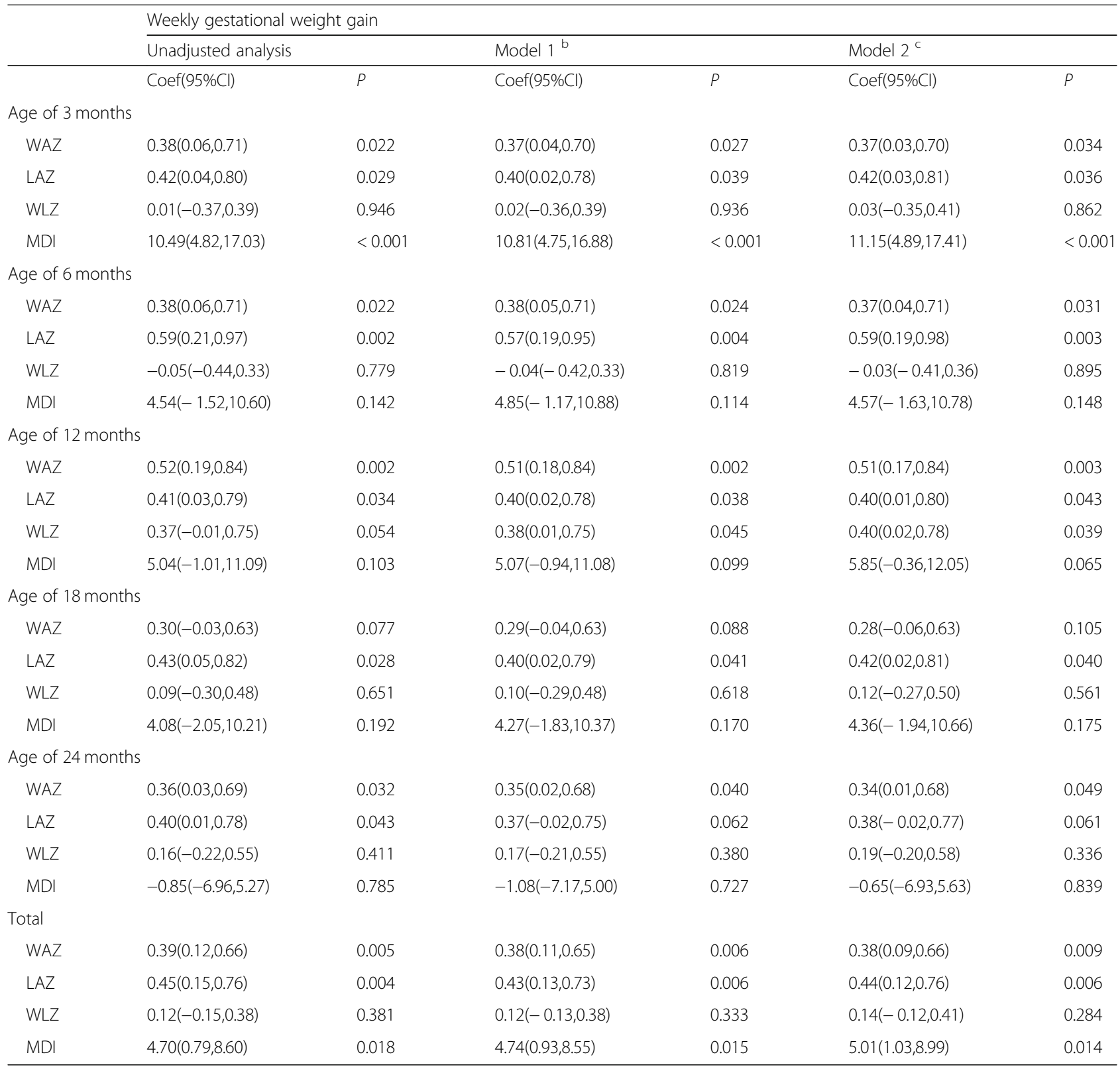

Abbreviation: CI Confidence interval, LAZ Length for age Z scores, MDI Mental development index, WAZ Weight for age Z scores

${ }^{a}$ Generalized estimated equation linear model were used to assess the association between weekly gestational weight gain and development of infants

${ }^{b}$ Model 1 include the variables of county, sex of infant, maternal age, educational level of parents, household wealth index, occupation of parents

'Model 2 include the variables of county, sex of infant, maternal age, educational level of parents, household wealth index, occupation of parents, gestational weeks at birth, gestational weeks when maternal weight measured, maternal pre-pregnancy BMI, type of prenatal micronutrient supplementation, doses of supplements consumed

mental development (MDI) tended to decline as the infant became older.

\section{Comparison with other studies}

Previous studies have reported the effect of maternal pre-pregnancy BMI on child development. For physical growth, evidence from a large cohort study conducted in Norway showed a positive association between the maternal pre-pregnancy BMI and offspring BMI at 3 years of age [32]. Results from an Iranian cross-sectional study showed the positive effects of pre-pregnancy body mass index on birth weight, where a low pre-pregnancy BMI was found to be a risk factor for low birth weight [33]. Another prospective pregnancy cohort study conducted in the United States found that a higher pre-pregnancy BMI was associated with higher WAZ and weight-for-length $\mathrm{z}$ scores of infants [7]. For mental development, a prospective study focusing on African-American children found 
that pre-pregnancy obese women were at risk of having children (mean age of 5.3 years) with diminished intellectual ability (general IQ and nonverbal scores) [34]. Evidence from another cohort of children born in Finland showed that pre-pregnancy maternal obesity $(\mathrm{BMI} \geq 30)$ was a risk factor for intellectual disability in adults aged 20 years, but a similar association was not found among children at the age of 11.5 years [35]. A large prospective cohort study in the United Kingdom found a negative effect of maternal pre-pregnancy BMI on children's cognitive performance at 5 and 7 years of age [9]. In the present study, the average effect of maternal pre-pregnancy BMI on the physical growth (WAZ and LAZ) of infants across different time points was identified. This result was consistent with the findings of other previous studies. An effect of maternal pre-pregnancy BMI on the mental development of infants was not found in the present study. However, the present study was conducted in poor areas of China where the incidence of maternal overweight or obesity is only $2.7 \%$, but the incidence of maternal underweight is $17.7 \%$. Conversely, the incidence of maternal overnutrition (overweight or obesity) and underweight in the UK are 29 and 5.4\%, respectively [9]. Therefore, the differences in the incidences of the maternal mothers who are underweight and over-nourished between poor areas in China and the UK may explain the non-significant effects of maternal pre-pregnancy BMI observed in the present study. Our present findings emphasize the importance of maternal pre-pregnancy nutritional status on the prevention of infants' undernutrition, especially in poor areas.

Many studies have also reported the importance of maternal weight gain during pregnancy on children's development. Specifically, many studies found an adverse effect of excessive or lower weight gain during pregnancy. Evidence from a cross-sectional study in Portugal showed that excessive gestational weight gain was significantly associated with a higher risk of overweight offspring in young school-aged children [36]. However, the recall bias of the Portuguese study could not be avoided due to the cross-sectional study design. A longitudinal study conducted in the United States reported that excessive gestational weight gain was associated with an increase in the offspring BMI $\mathrm{z}$-scores at 5 years of age [37]. Another US cohort study reported a similar association between the excessive gestational weight gain and the risk of children aged 7 years who were overweight [38]. Findings from a prospective study showed that inadequate gestational weight gain was significantly associated with increased odds of being small-for-gestational-age and decreased odds of postpartum weight retention. Excessive gestational weight gain was associated with a decreased risk of being small-for-gestational-age, increased odds of postpartum weight retention, and overweight children [39]. For mental development, a large prospective, population-based birth cohort study conducted in the United Kingdom reported that gestational weight gain was positively associated with IQ (at 8 years of age) and school entry assessment scores (at 4 years of age) [10]. Another birth cohort study in Poland reported that excessive gestational weight gain had a negative impact on the cognitive development of children at 7 years of age [40]. The results from the present study were similar to previous studies, as we found a positive and significant average effect of gestational weight gain on the physical growth and mental development of infants, and the effect of gestational weight gain on the physical growth was still significant at different time points of measurement. For the mental development of infants, the effect of maternal weekly gestational weight gain was only significant for the earlier period ( 3 months) of infants. In addition, because gestational weight gain was used as an indicator of nutritional status during pregnancy [41], an effect of the maternal pre-pregnancy weight was not found on the infants' mental development. Our results suggest the importance of improving prenatal nutritional status in the further development of children. Most previous studies categorized women as having gained excess, adequate or inadequate weight according to the Institute of Medicine guidelines [41], but the present study could not provide similar results as the times that maternal weight was measured in the first and third trimesters were not fixed.

To the best of our knowledge, most similar previous studies specified the effect of maternal BMI and weight gain on child development at certain ages, and the effect on child development at a specific period of time has rarely been reported, especially in poor areas. A repeated measures study design was used in the present study. Therefore, the average effect and time effect of maternal pre-pregnancy BMI and gestational weight gain could be comprehensively estimated. The present study found a positive effect of maternal pre-pregnancy BMI and gestational weight gain on the physical growth of infants. A possible explanation is that environmental factors, such as pre-pregnancy and prenatal lifestyle, including physical activity and food intake, which affect maternal BMI and gestational weight gain, were also reported to contribute to offspring growth [42]. These findings are consistent with activity interventions during pregnancy. For the mental development of infants, a significant association between the maternal pre-pregnancy BMI and MDI was not found at any age of the infants. A possible explanation for this could be the weak effect of pre-pregnancy nutritional status on the mental development of infants. Our sample was not large enough to 
detect such a small association between the maternal BMI and MDI. Our results showed that the effect of gestational weight gain on the MDI was only significant in infants at 3 months. In addition, the infants in the present study were followed until they reached an early school age, and a significant association between the gestational weight gain and mental development in the early school-aged children was not found (data not shown). Our previously published results showed the positive effect of prenatal multi-micronutrient supplementation on the mental development of infants at 1 year of age, but the association between the prenatal micronutrient supplementation and the mental development of early school-aged children was found to be nonsignificant. These results support the idea that the positive effects of prenatal nutrition on child mental development may fade over time. A possible explanation for this could be the present study was conducted in the poor areas in China. Evidence suggests that lower level of early educational exposure and nutritional advantages are available to rural infants as opposed to their urban counterparts [43, 44]. These postnatal factors that have been proved associated cognitive development of infants [45] might cover the effect of weekly gestational weight gain. In addition, recent animal studies have reported an association between the prenatal nutrition and mental development. Malnutrition appears to alter cell migration, cell numbers, synaptogenesis, myelinisation, neurotransmission and hippocampal formation in rats [46-49].

\section{Strengths and limitations}

The strengths of the study are its standardized measurement procedures and its prospective data collection. In addition, the repeated measures study design enabled us to comprehensively evaluate the average effect across different time points and the time effect of maternal BMI and gestational weight gain on child development. Our study also has several limitations. First, there were a few missing values in the variables of maternal weight and child development. After excluding the participants who were not weighted in the first trimester, we compared the baseline characteristics between the excluded and included groups. We found that the two groups did not differ in their baseline characteristics (Additional file 1: Table S1). In addition, we used a multiple imputation method as a sensitivity analysis, and we found that the results were robust after comparing the differences between the sensitivity analysis and the original analysis (Additional file 1: Tables S3 and S4). Second, we did not include all possible confounders. For example, we did not collect information of maternal dietary during pregnancy and lactation and parental mental development which is a strong factor of offspring mental development
[50] in addition to maternal psychological distress which may be associated with parenting behaviour. However, the results from a large cohort study showed that the significant association between maternal behavioural problems and offspring weight is not affected by the adjustment for maternal depression and anxiety [51]. Despite this, consistent outcomes after the adjustment for different confounders also proved present results to be reliable and robust. Although there were several limitations in the present study, this study contributes to the literature by providing robust data on the effects of maternal weight and weight gain in a poor area where there are concerns that inadequate in utero nutrition may have adverse effects on child development.

\section{Conclusion}

In conclusion, we identified the positive effect of pre-pregnancy BMI and gestational weight gain on the physical growth of infants. For the mental development of infants, the effect of weekly gestational weight gain was identified, but the effect appears to fade away over time. Our findings emphasized the importance of improving pre-pregnancy and prenatal nutrition on infants' development in a lower resource setting.

\section{Additional file}

Additional file 1: Table S1. Baseline characteristics of participants in excluded and included groups. Table S2. Association between gestational weekly weight gain and development of infants. Table S3. Association between maternal pre-pregnancy BMI and development of infant (sensitivity analysis, multiple imputation method). Table S4. Association between gestational weekly weight gain and development of infant (sensitivity analysis, multiple imputation method). (DOCX $33 \mathrm{~kb}$ )

\section{Abbreviations}

LAZ: Length for age Z scores; MDI: Mental development index; WAZ: Weight for age Z scores; WLZ: Weight for Length Z scores

\section{Acknowledgements}

We submit our thanks to the Health Departments of each project county, the local health bureaus, and education bureaus for their cooperation and organization of the field data collection. We also thank the staff of Xi'an Jiaotong University for their participation in the field data collection.

\section{Funding}

Supported by National Natural Science Foundation of China, 81230016; China Postdoctoral Science Foundation, 2016 M592804; New Century Excellent Talents in University grant, NCET-11-0417.

\section{Availability of data and materials}

The datasets used and/or analyzed during the current study are available from the corresponding author on reasonable request.

\section{Authors' contributions}

$\mathrm{CL}$ conceptualized and designed the research, drafted the initial manuscript, and approved the final manuscript as submitted. LZ, DW, SD, CT, WW carried out the initial data analyses, reviewed and revised the manuscript, and approved the final manuscript as submitted. HY was the principal investigator and designed the data collection instruments, coordinated and supervised data collection at the study sites, critically reviewed the manuscript, and approved the final manuscript as 
submitted. All authors approved the final manuscript as submitted and agree to be accountable for all aspects of the work.

\section{Ethics approval and consent to participate}

The study was approved by the Human Research Ethics Committee of Xi'an Jiaotong University Health Science Center.

\section{Consent for publication}

Not applicable.

\section{Competing interests}

The authors declare that they have no competing interests.

\section{Publisher's Note}

Springer Nature remains neutral with regard to jurisdictional claims in published maps and institutional affiliations.

\section{Author details}

${ }^{1}$ Department of Epidemiology and Biostatistics, School of Public Health, Xi'an Jiaotong University Health Science Center, Xi'an, China. ${ }^{2}$ Department of Clinical Sciences, Liverpool School of Tropical Medicine, Pembroke Place, Liverpool, UK. ${ }^{3}$ Nutrition and Food Safety Engineering Research Center of Shaanxi Province, Xi'an, China. ${ }^{4}$ Key Laboratory of Environment and Genes Related to Diseases, Xi'an Jiaotong University, Xi'an, China. ${ }^{5}$ School of Public Health, Xi'an Jiaotong University Health Science Center, No. 76 West Yanta Road, PO Box 46, Xi'an, Xi'an 710061, Shaanxi, China.

Received: 16 September 2018 Accepted: 14 January 2019 Published online: 23 January 2019

\section{References}

1. Wechsler D. The measurement and appraisal of adult intelligence. 4th ed. Baltimore: Williams \& Wilkins; 1958.

2. Piek JP, Dawson L, Smith LM, et al. The role of early fine and gross motor development on later motor and cognitive ability. Hum Mov Sci. 2008;27(5): 668-81.

3. Batty GD, Mortensen E, Nybo Andersen A-M, et al. Childhood intelligence in relation to adult coronary heart disease and stroke risk: evidence from a Danish birth cohort study. Paediatr Perinat Epidemiol. 2005;19:452-9.

4. Hart CL, Taylor MD, Davey Smith G, et al. Childhood IQ and cardiovascular disease in adulthood: prospective observational study linking the Scottish mental survey 1932 and the Midspan studies. Soc Sci Med. 2004;59:2131-8.

5. Gishti O, Gaillard R, Manniesing R, et al. Fetal and infant growth patterns associated with total and abdominal fat distribution in school-age children. The Journal of Clinical Endocrinology \& Metabolism. 2014;99(7):2557-66.

6. Javaid MK, Lekamwasam S, Clark J, et al. Infant growth influences proximal femoral geometry in adulthood. J Bone Miner Res. 2006;21(4):508-12.

7. Deierlein AL, Siega-Riz AM, Adair LS, et al. Effects of pre-pregnancy body mass index and gestational weight gain on infant anthropometric outcomes. J Pediatr. 2011;158(2):221-6.

8. Oken E, Kleinman KP, Belfort MB, et al. Associations of gestational weight gain with short-and longer-term maternal and child health outcomes. Am J Epidemiol. 2009;170(2):173-80.

9. Basatemur E, Gardiner J, Williams C, et al. Maternal prepregnancy BMI and child cognition: a longitudinal cohort study. Pediatrics. 2013;131(1):56-63.

10. Gage $\mathrm{SH}$, Lawlor DA, Tilling $\mathrm{K}$, et al. Associations of maternal weight gain in pregnancy with offspring cognition in childhood and adolescence: findings from the Avon longitudinal study of parents and children. Am J Epidemiol. 2013;177(5):402-10.

11. Zeng L, Dibley MJ, Cheng Y, Dang S, Kong L, Yan H. Impact of micronutrient supplementation during pregnancy on birth weight, duration of gestation, and perinatal mortality in rural western China: double blind cluster randomised controlled trial. BMJ. 2008;337:a2001.

12. Li C, Zeng $L$, Wang $D$, et al. Prenatal micronutrient supplementation is not associated with mental development of young school-aged children. J Nutr. 2015;145(8):1844-9.

13. Li Q, Yan $H$, Zeng $L$, et al. Effects of maternal multimicronutrient supplementation on the mental development of infants in rural western China: follow-up evaluation of a double-blind, randomized, controlled trial. Pediatrics. 2009;123(4):e685-92.
14. Anderson JW, Johnstone BM, Remley DT. Breast-feeding and cognitive development: a meta-analysis. Am J Clin Nutr. 1999;70:525-35.

15. Carpenter DO. Effects of metals on the nervous system of humans and animals. Int J Occup Med Environ Health. 2001;14:209-18.

16. World Health Organization. WHO child growth standards: length/height-forage, weight-for-age, weight-for-length, weight-for-height and body mass index-for-age: methods and development. Geneva: World Health Organization; 2006.

17. Bayley N. Bayley scales of infant development. 2nd ed. San Antonio, Tex: The Psychological Corporation; 1993.

18. Colombo J, Carlson SE. Is the measure the message: the BSID and nutritional interventions. Pediatrics. 2012;129(6):1166-7.

19. Van der Meulen BF, Ruiter SAJ, Lutje Spelberg HC, et al. BSID-II-NL Dutch manual 2002

20. Huang H, Tao SD, Zhang YW, et al. Standardization of Bayley scales of infant development in Shanghai. Chinese Journal of Child Health Care. 1993;1(3): 158-60.

21. Westera JJ, Houtzager BA, Overdiek B, et al. Applying Dutch and US versions of the BSID-II in Dutch children born preterm leads to different outcomes. Dev Med Child Neurol. 2008;50(6):445-9.

22. Fattah C, Farah N, Barry SC, et al. Maternal weight and body composition in the first trimester of pregnancy. Acta Obstet Gynecol Scand. 2010:89(7):952-5.

23. World Health Organization. Obesity: preventing and managing the global epidemic. Geneva, Switzerland: World Health Organization; 2000.

24. Li C, Zhu N, Zeng $L$, et al. Sex differences in the intellectual functioning of early school-aged children in rural China. BMC Public Health. 2016;16(1):288.

25. Christian P, Murray-Kolb LE, Khatry SK, et al. Prenatal micronutrient supplementation and intellectual and motor function in early school-aged children in Nepal. Jama. 2010;304:2716-23.

26. Li C, Zhu N, Zeng $L$, et al. Effect of prenatal and postnatal malnutrition on intellectual functioning in early school-aged children in rural western China. Medicine. 2016;95(31):e4161.

27. Adair LS, Fall CD, Osmond C, et al. Associations of linear growth and relative weight gain during early life with adult health and human capital in countries of low and middle income: findings from five birth cohort studies. Lancet. 2013;382(9891):525-34.

28. Igel U, Romppel M, Baar J, et al. Association between parental socioeconomic status and childhood weight status and the role of urbanicity. Public Health. 2016;139:209-11.

29. Schieve LA, Cogswell ME, Scanlon KS, et al. Prepregnancy body mass index and pregnancy weight gain: associations with preterm delivery. Obstet Gynecol. 2000;96(2):194-200

30. Lee KJ, Carlin JB. Multiple imputation for missing data: fully conditional specification versus multivariate normal imputation. Am J Epidemiol. 2010; 171(5):624-32

31. Spratt M, Carpenter J, Sterne JAC, et al. Strategies for multiple imputation in longitudinal studies. Am J Epidemiol. 2010;172(4):478-87.

32. Kopp US, Dahl-Jorgensen $\mathrm{K}$, Stigum $\mathrm{H}$, et al. The associations between maternal pre-pregnancy body mass index or gestational weight change during pregnancy and body mass index of the child at 3 years of age. Int J Obes. 2012;36(10):1325-32.

33. Yekta $Z$, Ayatollahi $H$, Porali $R$, et al. The effect of pre-pregnancy body mass index and gestational weight gain on pregnancy outcomes in urban care settings in Urmia-Iran. BMC Pregnancy and Childbirth. 2006;6(1):15.

34. Neggers YH, Goldenberg RL, Ramey SL, et al. Maternal prepregnancy body mass index and psychomotor development in children. Acta Obstet Gynecol Scand. 2003;82(3):235-40.

35. Heikura $U$, Taanila $\mathrm{A}$, Hartikainen $\mathrm{AL}$, et al. Variations in prenatal sociodemographic factors associated with intellectual disability: a study of the 20-year interval between two birth cohorts in northern Finland. Am J Epidemiol. 2008:167(2):169-77.

36. Moreira P, Padez C, Mourao-Carvalhal I, et al. Maternal weigh gain during pregnancy and overweight in Portuguese children. Int J Obes. 2007:31(4): 608-14

37. Hinkle SN, Sharma AJ, Swan DW, et al. Excess gestational weight gain is associated with child adiposity among mothers with normal and overweight prepregnancy weight status. J Nutr. 2012;142(10):1851-8.

38. Wrotniak BH, Shults J, Butts S, et al. Gestational weight gain and risk of overweight in the offspring at age $7 \mathrm{y}$ in a multicenter, multiethnic cohort study. Am J Clin Nutr. 2008;87(6):1818-24. 
39. Zilko CEM, Rehkopf D, Abrams B. Association of maternal gestational weight gain with short-and long-term maternal and child health outcomes. Am J Obstet Gynecol. 2010;202(6):574.e1-8.

40. Jedrychowski W, Perera F, Jankowski J, et al. Effect of exclusive breastfeeding on the development of children's cognitive function in the Krakow prospective birth cohort study. Eur J Pediatr. 2012;171(1):151-8.

41. Institute of Medicine and NRC. Weight gain during pregnancy: reexamining the guidelines. Washington, DC: The National Academies Press; 2009.

42. Zalbahar N, Mohamed HJBJ, Loy SL, et al. Association of parental body mass index before pregnancy on infant growth and body composition: evidence from a pregnancy cohort study in Malaysia. Obesity Research \& Clinical Practice. 2016;10:S35-47.

43. Sicular T, Ximing Y, Gustafsson B, et al. The urban-rural income gap and inequality in China. Rev Income Wealth. 2007;53(1):93-126.

44. Smith LC, Ruel MT, Ndiaye A. Why is child malnutrition lower in urban than in rural areas? Evidence from 36 developing countries. World Dev. 2005; 33(8):1285-305.

45. Liu J, Raine A, Venables P, et al. Malnutrition at age 3 years and lower cognitive ability at age 11: a prospective longitudinal study. Arch Pediatr Adolesc Med. 2003;157:593-600.

46. Nyaradi A, Li J, Hickling S, et al. The role of nutrition in children's neurocognitive development, from pregnancy through childhood. Front Hum Neurosci. 2013;7:97.

47. Alamy M, Bengelloun WA. Malnutrition and brain development: an analysis of the effects of inadequate diet during different stages of life in rat. Neurosci Biobehav Rev. 2012:36:1463-80.

48. Granados-Rojas L, Larriva-Sahd J, Cintra L, et al. Prenatal protein malnutrition decreases mossy fibers-CA3 thorny excrescences asymmetrical synapses in adult rats. Brain Res. 2002;933:164-71.

49. Mathangi DC, Namasivayam A. Effect of chronic protein restriction on motor co-ordination and brain neurotransmitters in albino rats. Food Chem Toxicol. 2001;39:1039-43.

50. Deary IJ, Strand S, Smith P, Fernandes C. Intelligence and educational achievement. Intelligence. 2007;35:13-21.

51. Alati R, Najman JM, O'Callaghan M, et al. Fetal growth and behavior problems in early adolescence: findings from the Mater University study of pregnancy. Int J Epidemiol. 2009:38:1390-400.

Ready to submit your research? Choose BMC and benefit from:

- fast, convenient online submission

- thorough peer review by experienced researchers in your field

- rapid publication on acceptance

- support for research data, including large and complex data types

- gold Open Access which fosters wider collaboration and increased citations

- maximum visibility for your research: over $100 \mathrm{M}$ website views per year

At $\mathrm{BMC}$, research is always in progress.

Learn more biomedcentral.com/submissions 